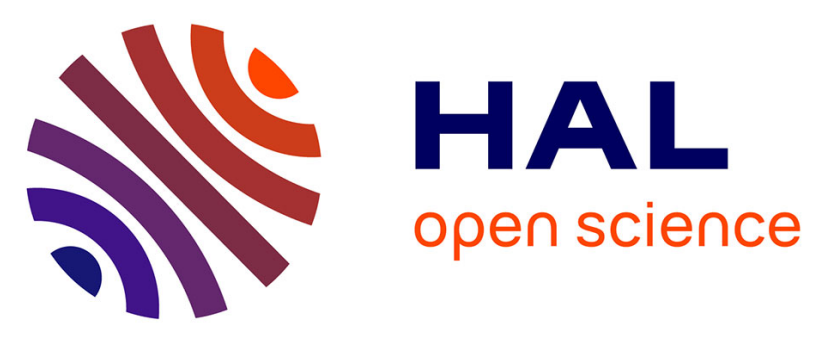

\title{
Multi-lane Vehicle-to-Vehicle Networks with Time-Varying Radio Ranges: Information Propagation Speed Properties
}

Emmanuel Baccelli, Philippe Jacquet, Bernard Mans, Georgios Rodolakis

\section{- To cite this version:}

Emmanuel Baccelli, Philippe Jacquet, Bernard Mans, Georgios Rodolakis. Multi-lane Vehicle-toVehicle Networks with Time-Varying Radio Ranges: Information Propagation Speed Properties. [Research Report] RR-8037, INRIA. 2012. hal-00722384

\section{HAL Id: hal-00722384 \\ https://hal.inria.fr/hal-00722384}

Submitted on 1 Aug 2012

HAL is a multi-disciplinary open access archive for the deposit and dissemination of scientific research documents, whether they are published or not. The documents may come from teaching and research institutions in France or abroad, or from public or private research centers.
L'archive ouverte pluridisciplinaire HAL, est destinée au dépôt et à la diffusion de documents scientifiques de niveau recherche, publiés ou non, émanant des établissements d'enseignement et de recherche français ou étrangers, des laboratoires publics ou privés. 
Multi-lane

Vehicle-to-Vehicle

Networks with

Time-Varying Radio

Ranges: Information

Propagation Speed

Properties

E. Baccelli, P. Jacquet , B. Mans , G. Rodolakis

RESEARCH

REPORT

$\mathrm{N}^{\circ} \mathbf{8 0 3 7}$

August 2012

Project-Team HiPERCOM 



\title{
Inzíán
}

\section{Multi-lane Vehicle-to-Vehicle Networks with Time-Varying Radio Ranges: Information Propagation Speed Properties}

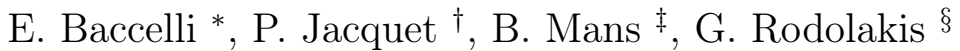 \\ Project-Team HiPERCOM
}

Research Report $\mathrm{n}^{\circ} 8037$ - August 2012 - 19 pages

\begin{abstract}
In this paper, we study the information propagation speed in multi-lane vehicle-tovehicle networks such as multi-lane roads or highways. We focus on the impact of time-varying radio ranges and of multiple lanes of vehicles, varying in speed and in density. We assess the existence of a vehicle density threshold under which information propagates on average at the fastest vehicle speed and above which information propagates dramatically faster. We first prove that no such phase transition occurs if there is only one lane, regardless of the density of vehicles, when one takes into account real-time radio communication range variations at the MAC layer. We then prove that, on the other hand, a phase transition exists as soon as there are multiple lanes with different vehicle speeds and appropriate densities. We characterize conditions under which the phase transition occurs and we derive bounds on the corresponding threshold as a simple relationship between the vehicle density on the fastest lane and the sum of the vehicle densities on the other lanes. Our results intrinsically encompass a wide range of vehicular network scenarios, including one-way and two-way roads, as well as special cases such as road side units and/or parked cars being used as relays. We confirm our analytical results using simulations carried out in several environments (ONE and Maple).
\end{abstract}

Key-words: DTN, Delay, Tolerant, Network, routing, epidemic, network, vehicular, protocol, radio, speed, propagation, information, multi-lane

* INRIA Saclay-ële-de-France

$\dagger$ Alcatel Lucent Bell Labs

$¥$ Macquarie University

$\S$ CERTH Information Technologies Institute

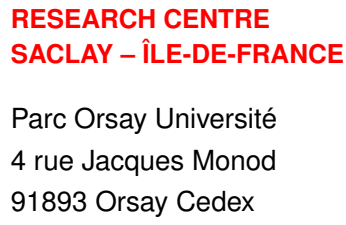




\section{Réseaux Véhiculaires Multi-Files avec Portées Radio Variables: Propriétés de la Vitesse de Propagation de l'Information}

Résumé : Ce document analyse les propriétés de la vitesse de propagation de l'information dans les réseaux véhiculaires multi-files avec portées radio variables.

Mots-clés : DTN, réseaux, véhiculaires, protocole, routage, épidémique, radio, vitesse, propagation, délai, tolérance, information, multi-files 


\section{Introduction}

In this paper, we study the information propagation speed in multi-lane vehicle-to-vehicle networks such as multi-lane roads or highways. We focus on the impact of multiple lanes of vehicles, varying in speed and in density. Large countries typically cover a great number of miles with multi-lane roads or highways, in part without telecommunication infrastructure. In the USA for instance, highways make about $75 \%$ of the total statute miles, and US roads have on average two lanes each way [14]. Our analysis shows the conditions under which a phase transition occurs in this context, concerning information propagation speed, with respect to the vehicle density. We prove that under a certain threshold, information propagates on average at the fastest vehicle speed, while above this threshold, information propagates significantly faster.

With applications such as safety on the road, ad hoc vehicular networks and vehicle-to-vehicle are receiving increasing attention (see recent surveys and challenges in [5, 6, 13]). Vehicular DTNs (Delay Tolerant Networks, i.e., networks in which end-to-end paths may not exist and communication routes may only be available through mobility and accumulation over time) have thus been considered in recent studies, and various analytical models have been proposed. Several general studies focus on characterizing the packet propagation delay in DTNs: in [8], the authors obtained analytical estimates of the upper bounds on the speed at which information can propagate in various models of multi-dimensional DTNs.

Other studies have more specifically targeted roads and vehicle-to-vehicle networks. In [11, the authors prove that the packet inter reception time distribution is a power-law and that long situation awareness black-outs are likely to occur in batch. In [15], the authors study vehicle traces and conclude that vehicles are very close to being Poisson distributed on highways. The authors of [4] also base themselves on traces gathered in DieselNET (the experimental vehicular network deployed by UMass) to elaborate and evaluate a novel vehicular routing algorithm. In [7], the authors provide a model for critical message dissemination in vehicular networks and derive results on the average delay in delivery of messages with respect to vehicle density. The authors of [17, propose an alternative model for vehicular networks and derived results on node connectivity, under the hypothesis that vehicles are exponentially distributed. The study is based on queuing theory techniques and characterizes the relationship between node connectivity and several parameters including speed distribution and traffic flow. In [16, the authors model vehicles on a highway, and study message propagation among vehicles in the same direction, taking into account speed differences between vehicles, while in [12] authors study message dissemination among vehicles in opposing directions and conclude that using both directions increases dissemination significantly.

Studies such as [1, 2] focus on information propagation speed in one-dimensional vehicleto-vehicle with constant speeds in each direction. In [1 the authors introduce a model based on space discretization to derive upper and lower bounds in the highway model under the assumption that the radio propagation speed is finite. Their bounds, although not converging, clearly indicates the existence of a phase transition phenomenon for the information propagation speed. Comparatively in [2], the authors introduce a model based on Poisson point process on continuous space, that allows both infinite and finite radio propagation speed, and derive more fine-grained results above and below the threshold. Using the model, the authors prove and explicitly characterize the phase transition. In [18], the authors derive equations for the expected value and variance of propagation distance through inter-vehicle communication when the distance between vehicle follows a general distribution. The paper also provide simple equations characterizing the expected number of vehicles covered and the probability distribution of the distance over which the information is propagated.

Studies such as [19, 20] are the closest related work to our study. In [20], the authors consider 
a one-dimensional vehicle-to-vehicle network with unit-disk radio range where all vehicle travel in the same direction, with random speeds following a Gaussian distribution. The paper derives the impact of various parameters on information propagation speed. The authors extend their results in $[19$ to a multi-stream case where vehicles in different streams have different speed distributions and derived analytical formulas. Comparatively, we here consider a model accommodating timevarying, possibly unbounded, radio ranges for vehicles on multi-lane roads, where vehicles on a particular lane all have the same speed. Since we consider the speed of vehicles only relatively to the speed of vehicles on the fastest lane, our model naturally encompasses both cases of one-way and two-way roads, as well as special cases such as road side units and/or parked cars being used as relays (e.g., [10]). The goal of this paper is to increase our understanding of the fundamental performance limits of mobile vehicle-to-vehicle networks with time-varying radio ranges and to thus complement the above-cited works.

In this context, our contributions are as follows: (1) in Section 2 we present a time-varying radio range model for information propagation in multi-lane vehicle-to-vehicle networks that takes into account real-time radio communication range variations at the MAC layer (such as Aloha in the Poisson shot model, e.g., [3]) and list our corresponding results for all cases of number of lanes; (2) we prove that no such phase transition occurs concerning the information propagation speed with respect to the vehicle densities if there is only one lane, regardless of the car densities in Section 3, and (3) we prove that, on the other hand, a phase transition exists as soon as there is a second lane with different vehicle speed and sufficient density in Section 4. We characterize the phase transition threshold in this context, and we prove that there are still cases where no such phase transition occurs; (4) in Section 5. we focus on information propagation speed when there are three lanes or more. We show that under certain conditions a phase transition exists and we derive bounds on the threshold as a simple relationship between the vehicle density on the fastest lane and the sum of the vehicle densities on the other lanes. We also prove that that there are still cases where no such phase transition occurs; (5) Finally we validate the provided analysis with simulations in multiple environments (The One and Maple), in Section 6.

\section{$2 \quad$ Model and Results}

\section{1 $\quad$ Model}

We consider a highway with multiple lanes of vehicles at fixed speed, numbered 0 to $k$ : on lane $i$, the vehicle speed is $v_{i}$. The vehicle density on lane $i$ is Poisson of intensity $\lambda_{i}$. Vehicle speeds are in decreasing order: lane 0 is called the fast lane, determined as the lane of vehicles with the fastest eastbound speed. Lanes from 1 to $k$ are conversely called the slow lanes. In the following, we consider vehicle speeds in the referential of the vehicles of the fast lane.

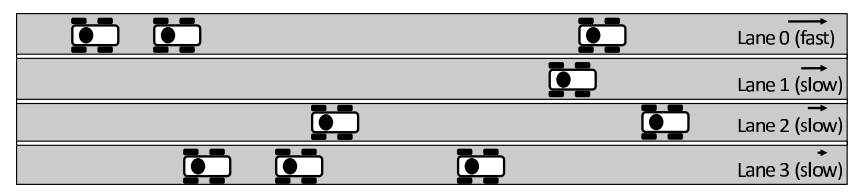

Figure 1: Vehicular network on a highway with four lanes of identical direction.

For instance, in Figure 1, we depict a vehicular network of four eastbound lanes; lanes are numbered in decreasing speed order. In Figure 2, we depict an example of a four-lane highway, 


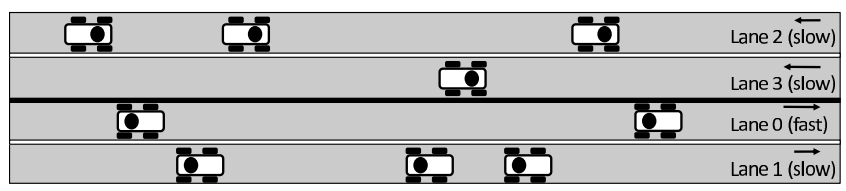

Figure 2: Vehicular network on a bidirectional highway with four lanes.

with two eastbound and two westbound lanes. Thus, we number lanes in decreasing eastbound speed: 0 . fast eastbound lane, 1. slow eastbound lane, 2. slow westbound lane, and 3. fast westbound lane. We note that, once we have defined the lane numbering, the actual directions of the lane speeds do not matter anymore in our model. Note that this also allows to account for static lanes where cars are parked along the road. As we consider the speeds in the referential of the fast lane, the speed of lane 0 will be 0 , and the speeds of the remaining lanes will be negative.

The radio propagation, as well as the store-and-forward time are considered instantaneous. For radio transmissions, we will consider alternatively (i) a fixed radio range, or, more realistically, (ii) a time-varying radio range.

In the former case (i), we will assume that the radio range of each transmission is of length $R$ in each direction (e.g., $R=1$ for all vehicles, all the time).

In the latter case (ii), we suppose that the radio range $R$ varies in time in a random way and is unbounded (i.e., for any distance $x, P(R>x) \neq 0$ ). We suppose that each vehicle transmits according to a time Poisson process of intensity $\nu>0$, with a random range $R$. We assume the following properties concerning the random variable $R$. Let $\zeta$ be a complex quantity with the dimension of inverse distance. For all $\zeta$,

1. $f(\zeta)=E\left(e^{-\zeta R}\right)$ is defined and is not infinite;

2. for all $x, E\left(e^{-(R-x) \zeta} \mid R>x\right)$ is defined and bounded by some function $g(\zeta)$.

The first condition implies that, $\lim _{x \rightarrow \infty} e^{\alpha x} P(R>x)=0$, for all $\alpha>0$. It is satisfied, e.g., when there exist $D>0$ and $\delta>1$, such that $P(R>x) \sim \exp \left(-D x^{\delta}\right)$. The second condition is satisfied when, for all $x$, the random variable $R-x$, under the condition that $R>x$, is smaller or equal in probability to a certain random variable $\bar{R}$, such that $E\left(e^{-\zeta \bar{R}}\right)$ is defined; in this case, we can take $g(\zeta)=E\left(e^{-\zeta \bar{R}}\right)$.

In particular, these conditions are satisfied for slotted Aloha in the Poisson shot noise model 3]. For example, in a one-dimensional highway network, when the power attenuation is of pathloss exponent $2, P(R>x)$ is of the form $e^{-x^{2}}$. Note, however, that we are not studying the Aloha protocol over the considered vehicle-to-vehicle network. Indeed, Aloha would imply a correlation between the radio ranges and the actual positions of the cars. In contrast, our model assumes independence between radio ranges and car positions.

Without loss of generality, we focus on the propagation of information in the eastbound direction. Our aim is the evaluation of the maximum speed at which a packet of information can propagate. An information packet propagates in the following manner, illustrated in the case of a two-lane highway in Figure 3 it moves toward the east jumping from car to car until it stops because the next car is beyond radio range. The information packet waits on the right-most car until either a transmission of large enough radio range occurs to move forward, or the gap is filled by cars of other lanes (in the case of fixed range, this is the only way for the information to propagate further). 


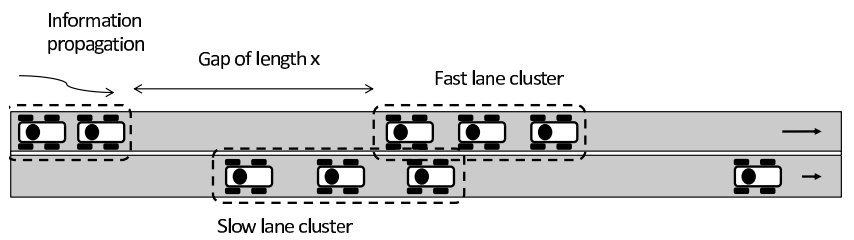

(a)

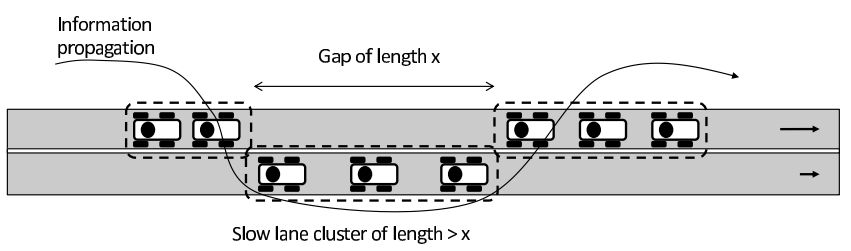

(b)

Figure 3: Eastbound information propagation: information is carried by the rightmost fast-lane car before the gap (a), until either a transmission of sufficiently large range occurs (in the variable range case), and/or the gap is bridged by slow-lane cars so that the information can propagate further (b).

We denote $L(t)$ the distance traveled by the information during time $t$ in the fast lane. We consider the distance traveled with respect to the referential of the fast-lane cars. We define the average information propagation speed $v_{p}$ as:

$$
v_{p}=\lim _{t \rightarrow \infty} \frac{\mathbf{E}(L(t))}{t}
$$

\section{$2.2 \quad$ Results}

The main results presented in this paper are the proofs of the following five theorems, corresponding to various cases of number of lanes: one (Theorem 1), two (Theorems 2 and 3), and three or more lanes (Theorems 4 and 5). For all five theorems, we analyze the asymptotic average information propagation speed $v_{p}$ (without loss of generality, in the referential of the vehicles of the fastest eastbound lane).

Our first theorem proves that for a single one-way lane, the information propagation speed is limited to the speed of the fastest car, regardless of the vehicle density $\lambda_{0}$.

Theorem 1. In a vehicular network with a single (one-way) lane where the radio range varies, the average asymptotic information propagation speed $v_{p}$ with respect to the referential of the cars is null, regardless of the vehicle density $\lambda_{0}$.

We then consider two-lane networks (i.e., the fastest vehicle lane's density is Poisson with intensity $\lambda_{0}$, while the slower vehicle lane's density is Poisson with intensity $\lambda_{1}$ ). We prove that, depending of the densities, there is a phase transition concerning the information propagation speed $v_{p}$ : there are cases where the average asymptotic information propagation speed is above the speed of the fastest car (Theorem 2), and others where it is limited to the speed of the fastest car (Theorem 3). We also characterize the corresponding threshold. For the remainder of the 
paper, for $x>0$, we denote $x^{*}$ the conjugate of $x$ with respect to the function $x e^{-x}: x^{*}$ is the alternate solution of the equation $x^{*} e^{-x^{*}}=x e^{-x}$. Notice that $x^{* *}=x$ and $1^{*}=1$.

Theorem 2. Consider a vehicular network with two lanes, where the radio range $R$ varies, with respective car densities $\lambda_{0}$ and $\lambda_{1}$, and respective speeds $v_{0}$ and $v_{1}$. Let a distance $r_{0}>0$ such that $P\left(R \geq r_{0}\right) \neq 0$. For all $0<a<1 / 2$, and for $\rho=-\log \left(1-\exp \left(-P\left(R \geq r_{0}\right) \frac{\nu a}{v_{0}-v_{1}}\right)\right)$, the average asymptotic information propagation speed $v_{p}$ with respect to the referential of the fastest cars is strictly larger than 0 , when $r_{0} \lambda_{0}>\frac{1}{1-2 a}\left(2 \rho+\left(r_{0} \lambda_{1}\right)^{*}\right)$.

However, we also have the equivalent of Theorem 11 s result for two lanes of low densities.

Theorem 3. Consider a vehicular network with two lanes, where the radio range $R$ varies, with respective car densities $\lambda_{0}$ and $\lambda_{1}$, and respective speeds $v_{0}$ and $v_{1}$. There exists $\rho_{0}>0$, which is a function of $\left(v_{0}-v_{1}\right)$ and $\lambda_{1}$, such that, when $\lambda_{0}<\rho_{0}$, the average asymptotic information propagation speed $v_{p}$ with respect to the referential of the fastest cars is null.

The last two theorems complete the analysis by considering three lanes or more. We first prove that there are cases where the average asymptotic information propagation speed is above the speed of the fastest car (Theorem 4), and characterize the corresponding threshold. Secondly, we prove that there exist non-null densities for which the average asymptotic information propagation speed is limited to the speed of the fastest car (Theorem 5). (Note that this case is different from Theorem 3, as it would be necessary to have an empty third lane, i.e., with a density $\lambda_{i}=0$.)

Theorem 4. Consider a vehicular network with three or more lanes, numbered 0 to $k$, where the radio range varies. For all $0<a<1 / 2$, there exists a distance $r_{0}>0$ and a scalar $\rho>0$, such that the average asymptotic information propagation speed $v_{p}$ with respect to the referential of the fastest cars (lane 0) is strictly larger than 0 , when $r_{0} \lambda_{0} \geq \frac{1}{1-2 a}\left(2 \rho+\left(r_{0} \sum_{i=1}^{i=k} \lambda_{i}\right)^{*}\right)$.

Theorem 5. Consider a vehicular network with three or more lanes, numbered 0 to $k$. There exists a tuple of car densities, such that $\forall i: \lambda_{i} \neq 0$, for which the average information propagation speed $v_{p}$ is null.

\section{Single Lane: Proof of Theorem 1}

As the information propagation speed is considered in the referential of the vehicles (say, in eastbound direction), we can equivalently assume that the cars are fixed, and consider the information propagation speed $v_{p}$ toward the east, solely based on the transmissions. We recall that the emission process of the vehicles is a time Poisson process of intensity $\nu>0$, with a random radio range $R$.

We call the rightmost car that has received the information at a given time $t$, the current car at time $t$. Without loss of generality, we consider that only the current car transmits the information; or, similarly, we can assume that the random radio range process includes the overlap from the ranges of previous cars to the left, if they participate in the transmission.

In the following, we describe a process to provide an upper bound on the information propagation speed $\bar{v}_{p}$. If the gap from the current car to the next car to the right is $x$, then one has to wait that the radio range exceeds $x$, before the information reaches the next car. When the radio range $R$ exceeds $x$, all the cars that are located between $x$ and $R$ receive the information. The speed upper-bound process consists in assuming that the next current car is located exactly at the range limit, i.e. at distance $R$ from the previous current car. 
Therefore, under the upper bound model, the gaps $x$ between the current cars and the following cars are i.i.d. random exponential variables, with rate $\lambda$. Given that the gap is of length $x$, the time $T(x)$ needed to move the message to the next current car satisfies:

$$
E(T(x))=\frac{1}{\nu P(R>x)},
$$

and the distance $D(x)$ traveled by the information at this time satisfies:

$$
E(D(x))=E(R \mid R>x) .
$$

Since this is a memoryless process, the average information propagation speed upper-bound $\overline{v_{p}}$ is (denoting $\lambda_{0}=\lambda$ ):

$$
\bar{v}_{p}=\frac{\int_{0}^{\infty} E(D(x)) \lambda e^{-\lambda x} d x}{\int_{0}^{\infty} E(T(x)) \lambda e^{-\lambda x} d x} .
$$

From the condition $\lim _{x \rightarrow \infty} e^{\lambda x} P(R>x)=0$, we get $\int_{0}^{\infty} E(T(x)) \lambda e^{-\lambda x} d x=\infty$. From the condition $E(R \mid R>x) \leq x+E(\bar{R})$, we have $\int_{0}^{\infty} E(D(x)) \lambda e^{-\lambda x} d x \leq \frac{1}{\lambda}+E(\bar{R})<\infty$. Therefore, the average information propagation speed $v_{p}$ is null.

\section{Two Lanes}

\subsection{Proof of Theorem 2}

For the purpose of the proof, we define a lower bound process on the information propagation speed. Let $r_{0}>0$ be such that $P\left(R \geq r_{0}\right) \neq 0$. We define the lower-bound process by bounding the radio range $R$ with a new range $R^{\prime}$, as follows:

- when $R \geq r_{0}$, then $R^{\prime}=r_{0}$;

- when $R<r_{0}$, then $R^{\prime}=0$.

We consider the information propagation on the fast (say eastbound) lane. In the lower bound model, cars must be separated by less than $r_{0}$, in order to be able to communicate. We call $r_{0}$-cluster a set of consecutive cars such that two consecutive cars are separated by distance less than $r_{0}$. We call length of a cluster the distance between the first and the last car of a cluster. A recursive definition of an $r_{0}$-cluster, spreading eastwards from position $y$, namely $\mathcal{C}_{r_{0}}(y)$, is:

$$
\mathcal{C}_{r_{0}}(y)=\mathcal{I}_{r_{0}}(y) \cup \mathcal{C}_{r_{0}}(z),
$$

where $\mathcal{I}_{r_{0}}(y)$ is the set of cars located in the segment $\left[y, y+r_{0}\right]$, and $z$ is the position of the rightmost car in $\mathcal{I}_{r_{0}}(y)$.

Therefore, if the current car on lane 0 (the fast lane) is separated from the next car on lane 0 by a gap of length $r_{0}(1+x)$, for $x>0$, then it needs to wait for a slow lane cluster of length at least $r_{0} x$ to bridge the gap.

The number of retransmissions of radio range $r_{0}$, to cover a cluster of length $r_{0} x$, cannot exceed $\lceil 2 x\rceil$. Let $a>0$ be a real number, and assume that the time between two retransmissions is smaller than $a \frac{r_{0}}{v_{0}-v_{1}}$. The latter occurs with probability $e^{-\rho}=1-\exp \left(-P\left(R \geq r_{0}\right) \nu a \frac{r_{0}}{v_{0}-v_{1}}\right)$, for some $\rho>0$ (since, we recall that the node emission process is a Poisson process of intensity $\nu>0$ ). This also implies that the cluster of length $x r_{0}$ travels a distance smaller than $\lceil 2 x\rceil a r_{0}$. Assuming now that $a<1 / 2$, a cluster of length $\frac{x r_{0}}{1-2 a}$ would suffice to bridge a gap of length 
$r_{0}(1+x)$, provided that successive retransmissions are all separated by less than $a \frac{r_{0}}{v_{0}-v_{1}}$ time units (which occurs with probability greater than $e^{-\rho 2 x /(1-2 a)}$ ).

From [2, equation (10)], with a lane density normalized by the radio range, we know that the average time to get an $r_{0}$-cluster of length $y r_{0}$ is $\Theta\left(e^{\left(r_{0} \lambda_{1}\right)^{*} y}\right)$, as expressed in the following lemma.

Lemma 1. The average distance to a car on the slow lane, which begins an $r_{0}$-cluster of slow lane cars of length larger than $r_{0} x$, is $\Theta\left(e^{\left(r_{0} \lambda_{1}\right)^{*} x}\right)$, when $x \rightarrow \infty$.

Therefore, the time needed to get an $r_{0}$-cluster of length $r_{0} y$, where all successive retransmissions are separated by less than $a \frac{r_{0}}{v_{0}-v_{1}}$ time units, is $\Theta\left(e^{\left(2 \rho+\left(r_{0} \lambda_{1}\right)^{*}\right) y}\right)$. Using the methodology in [2, Section III-A, eq. (9)], the average distance to such a cluster on the slow lane is $\Theta\left(\exp \left(\frac{2 \rho+\left(r_{0} \lambda_{1}\right)^{*}}{1-2 a} x\right)\right)$, and thus the time $T^{\prime}(x)$ needed to bridge a gap of length $r_{0}(1+x)$ is:

$$
E\left(T^{\prime}(x)\right)=\Theta\left(\exp \left(\frac{2 \rho+\left(r_{0} \lambda_{1}\right)^{*}}{1-2 a} x\right)\right)
$$

The distance $D^{\prime}(x)$ traveled by the information (in the referential of fast-lane cars) satisfies:

$$
E\left(D^{\prime}(x)\right) \geq r_{0}(1+x)
$$

Therefore, the information propagation speed $v_{p}$ is:

$$
\begin{aligned}
v_{p} & \geq \frac{\int_{0}^{x} E\left(D^{\prime}(x)\right) \lambda_{0} r_{0} e^{-\lambda_{0} r_{0} x} d x}{\int_{0}^{x} E\left(T^{\prime}(x)\right) \lambda_{0} e^{-\lambda_{0} r_{0} x} d x} \\
& \geq \frac{r_{0}+1 / \lambda_{0}}{\int_{0}^{x} E\left(T^{\prime}(x)\right) \lambda_{0} r_{0} e^{-\lambda_{0} r_{0} x} d x} .
\end{aligned}
$$

It turns out that the integral in the denominator converges when $r_{0} \lambda_{0}>\frac{2 \rho+\left(r_{0} \lambda_{1}\right)^{*}}{1-2 a}$ and, with this condition, $v_{p}>0$.

\subsection{Proof of Theorem 3}

See appendix.

\section{$5 \quad$ Three or more Lanes}

\subsection{Proof of Theorem 4}

We take the framework of the proof of Theorem 2, with $r_{0}$ such that $P\left(R>r_{0}\right) \neq 0$. We will first consider the case where $\nu \rightarrow \infty$, i.e., when the channel access delay $\frac{1}{\nu}$ tends to 0 . In this case, we have $\rho=a=0$, and we are in the framework of [2] with a unit disk graph model, but with the difference that instead of 1 , the range here is $r_{0}$.

To simplify the notation, we denote $\Lambda_{s}=\sum_{i=1}^{i=k} \lambda_{i}$. We consider that the information is blocked by a gap of length $r_{0}(1+x)$ on the fast lane. The aim is to give an upper bound of the time needed to have an $r_{0}$-cluster of length at least $r_{0} x$, made of cars on slow lanes, which covers the gap. But, since clusters are not permanent in time, and experience continuous changes due to the differences of car motions, we will consider here instantaneous $r_{0}$-clusters. A recursive 
definition of an instantaneous $r_{0}$-cluster, spreading eastwards from position $y$ and at time $t$, namely $\mathcal{C}_{r_{0}}(y, t)$, is:

$$
\mathcal{C}_{r_{0}}(y, t)=\mathcal{I}_{r_{0}}(y, t) \cup \mathcal{C}_{r_{0}}(z, t),
$$

where $\mathcal{I}_{r_{0}}(y, t)$ is the set of cars located in the segment $\left[y, y+r_{0}\right]$ at time $t$, and $z$ is the position of the rightmost car in $\mathcal{I}_{r_{0}}(y, t)$ at time $t$.

We use a version of lemma 1 adapted to the case where the cars on slow lanes have relative motions. The following is directly derived from [2, Lemma 2].

Lemma 2. The probability that a given car, at a given time, begins an instantaneous $r_{0}$-cluster, of slow lane cars of length longer than $r_{0} x$, is $\Theta\left(e^{-\left(r_{0} \Lambda_{s}\right)^{*} x}\right)$, when $x \rightarrow \infty$.

The main point now is to track the correlation between cars and time, since cars move relatively to one another. To this end, we consider the process of cluster formation that starts with a car on lane 1, the fastest among the slow lanes. Without loss of generality, we assume that $\lambda_{1} \neq 0$.

We place ourselves in the referential of cars on lane 1 . We consider a road segment $[A, B)$ in this referential, for some $A<B$. Since the cars on the other slow lanes have a relative westbound motion, and since Poisson processes are memoryless, the events in the interval $[A, B)$, at a given time $t$, and the future events in the interval $[B, \infty)$ are independent.

To make use of this property for instantaneous cluster formations, we split the slowest lane $k$ into segments of length $(1+x) r_{0}$, numbered from 1 and onwards.

We concentrate on the first segment. Let us consider the first car in lane 1 . The probability that this lane 1 car starts a slow lane $r_{0}$-cluster, of length smaller than $x r_{0}$, at the time when this lane 1 car arrives in range of the current car on lane 0 , is smaller than $1-C e^{-\left(r_{0} \Lambda_{s}\right)^{*} x}$ (with $C>0$, as deduced from Lemma 2). Similarly, if we include the probability that no car of lane 1 is present in this segment, $\left(i . e ., e^{-(1+x) r_{0} \lambda_{1}}\right)$, then the probability that there is no instantaneous $r_{0}$-cluster, starting in the first segment, would have a length greater than $r_{0} x$ when in range with the current car, is smaller than $1-C\left(1-e^{-(1+x) r_{0} \lambda_{1}}\right) e^{-\left(r_{0} \Lambda_{s}\right)^{*} x}$.

Since this event only depends on the car positions in the first and second segments when the first car arrives in range of the current car, this event is independent of what will happen in the next segments. Thus, the apparitions of further $r_{0}$-clusters of length smaller than $r_{0} x$ on odd segments are i.i.d.

The average number of segments, before the formation of an instantaneous $r_{0}$-cluster of length greater than $r_{0} x$, is smaller than:

$$
\sum_{n \geq 0} 2\left(1-\left(1-e^{-(1+x) r_{0} \lambda_{1}}\right) C e^{-\left(r_{0} \Lambda_{s}\right)^{*} x}\right)^{n}=2 \frac{\exp \left(\left(r_{0} \Lambda_{s}\right)^{*} x\right)}{C\left(1-e^{-r_{0} \lambda_{1}}\right)} .
$$

Therefore, the time $T^{\prime}(x)$, before the gap of length $(1+x) r_{0}$ is bridged by an instantaneous cluster made of slow lane clusters, satisfies the inequality:

$$
E\left(T^{\prime}(x)\right) \leq \frac{2(x+1) r_{0}}{v_{0}-v_{1}} \frac{\exp \left(\left(r_{0} \Lambda_{s}\right)^{*} x\right)}{\left(1-e^{-r_{0} \lambda_{1}}\right) C}
$$

Thus, this average time $\int_{0}^{\infty} E\left(T^{\prime}(x)\right) \lambda_{0} r_{0} e^{-x r_{0} \lambda_{0}} d x$ is finite as soon as $r_{0} \lambda_{0}<\left(r_{0} \Lambda_{s}\right)^{*}$. This terminates the proof for $\nu \rightarrow \infty$. Notice again that, in this case, $\rho=a=0$.

When $\nu<\infty$, we cannot use the same approach: indeed, in this case, the time between retransmissions does not allow us to work with instantaneous clusters. In this case, we must consider a refined version of Lemma 2, where instantaneous $r_{0}$-clusters are replaced by $\tau$-timed $r_{0}$-clusters, i.e., $r_{0}$-clusters obtained with retransmissions delayed each by $\tau$ seconds exactly. A 


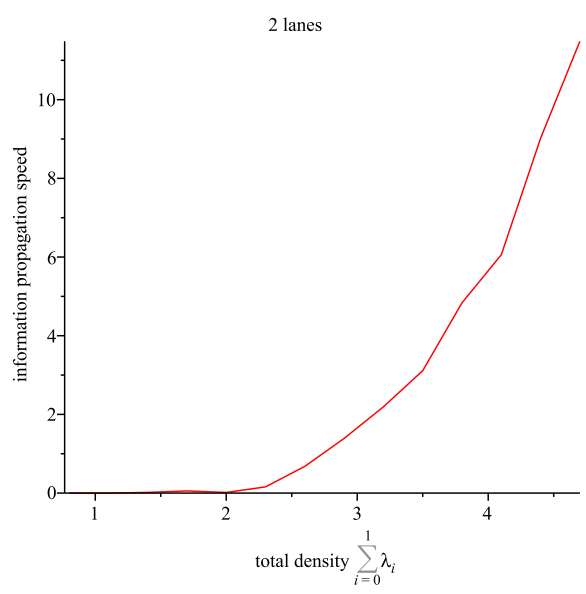

Figure 4: Maple simulations: 2 lanes, fixed radio range. Information propagation speed $v_{p}$ for $\lambda_{0}=\lambda_{1}$, versus the total vehicle density $\lambda_{0}+\lambda_{1}$.

recursive definition of an instantaneous $\left(r_{0}, \tau\right)$-cluster, spreading eastbound from position $y$ and at time $t$, namely $\mathcal{C}_{r_{0}, \tau}(y, t)$, is:

$$
\mathcal{C}_{r_{0}, \tau}(y, t)=\mathcal{I}_{r_{0}}(y, t) \cup \mathcal{C}_{r_{0}, \tau}(z, t+\tau),
$$

where $z$ is the position of the rightmost car in $\mathcal{I}_{r_{0}}(y)$ at time $t+\tau$. We have the following lemma, equivalent to lemma 2 .

Lemma 3. Let $\tau>0$, the probability that a given car, at a given time, begins an instantaneous $\tau$-timed $r_{0}$-cluster of slow lane cars of length larger than $r_{0} x$ is $\Theta\left(e^{-\left(r_{0} \Lambda_{s}\right)^{*} x}\right)$, when $x \rightarrow \infty$.

The end of the proof is only sketched, as it follows an approach similar to Theorem 2, We define $\tau=a \frac{r_{0}}{v_{0}-v_{1}}$ for some $a>0$ small enough, and we consider timed clusters larger than $(1+x) r_{0} \frac{1}{1-2 a}$, ensuring they can actually bridge a gap of length $(1+x) r_{0}$. We then consider $\left(r_{0}-\varepsilon\right)$-clusters instead of $r_{0}$-clusters, in order to cope with the small local modification of the clusters, since the retransmissions are not exactly after a time $\tau$, but within a time $\tau$ (we take $\left.\varepsilon=\tau\left(v_{1}-v_{k}\right)\right)$.

\subsection{Proof of Theorem 5}

See appendix.

\section{Simulations}

\subsection{Maple Simulations}

In this section we compare the theoretical analysis with numerical simulations performed using Maple. In this case, the simulations follow precisely the highway model described in Section 2 ; we generate Poisson traffic on multiple lanes, each moving at constant speed.

First, we consider the fixed-range scenario, where the radio transmission range is $R=1$ (that is, we normalize our parameters based on the radio range unit), and radio transmissions are instantaneous. We simulate a highway which is sufficiently large to provide either: a large number 


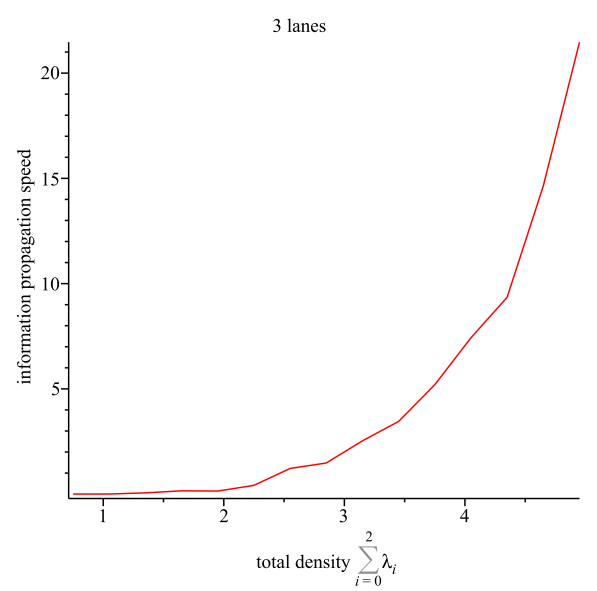

Figure 5: Maple simulations: 3 lanes, fixed radio range. Information propagation speed $v_{p}$ for $\lambda_{0}=\lambda_{1}=\lambda_{2}$, versus the total vehicle density.

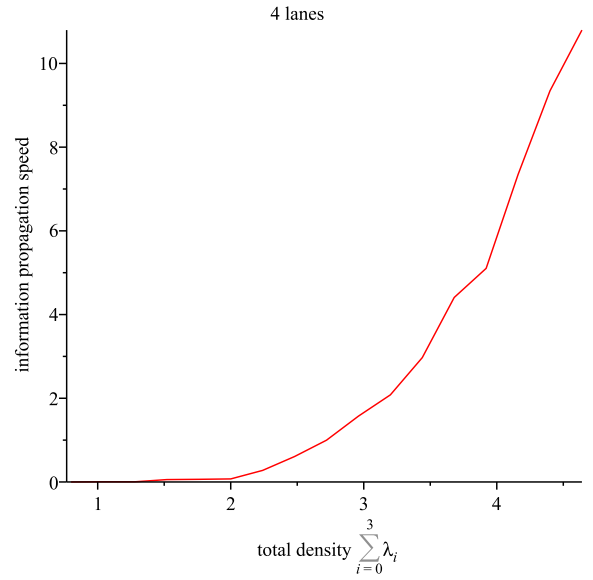

Figure 6: Maple simulations: 4 lanes, fixed radio range. Information propagation speed $v_{p}$ for $\lambda_{0}=\lambda_{1}=\lambda_{2}=\lambda_{3}$, versus the total vehicle density.

of bridging operations (of order at least $10^{3}$ ) for all considered traffic densities, or a large number of clusters in each lane (of order at least $10^{4}$ ), for the situation below the phase transition threshold where the information propagation speed might be equal to the fast-lane speed, according to the analysis. We measure the information propagation speed which is achieved using optimal store-carry-forward epidemic routing, by taking the ratio of the total propagation distance over the corresponding delay, and averaging over multiple iterations of randomly generated traffic. We vary the total traffic density, and we plot the resulting information propagation speed. Figures 4.5 and 6 show the evolution of the information propagation speed versus the total vehicle density, when $\lambda_{i}=\lambda_{0}$, for all $i$, for two, three and four lanes, respectively. The fast lane speed is assumed to be 0 (as we measure the speed in the referential of the fast cars). In Figure 4 , the slow lane speed is 0.4 radio range per second. In Figure 5 the slow lane speeds are 0.4 radio range per second and 0.6 radio range per second. In Figure 6, the slow lane speed are 0.4 


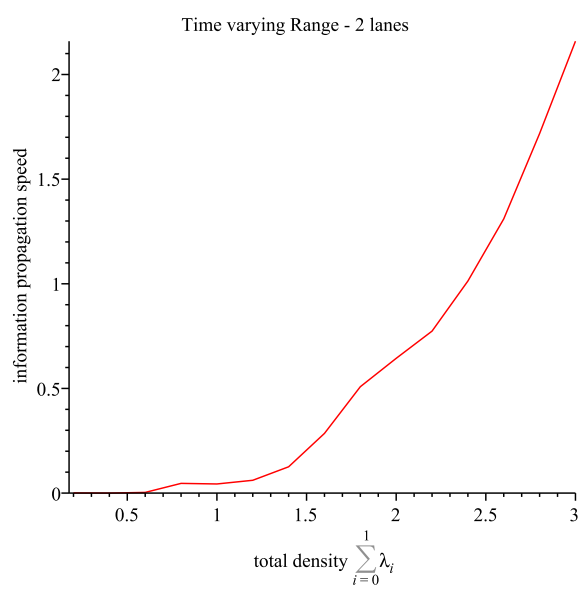

Figure 7: Maple simulations: 2 lanes, varying radio range $\left.\left(P(R>x)=e^{-x^{2}}\right)\right)$. Information propagation speed $v_{p}$ for $\lambda_{0}=\lambda_{1}$, versus the total vehicle density $\lambda_{0}+\lambda_{1}$.

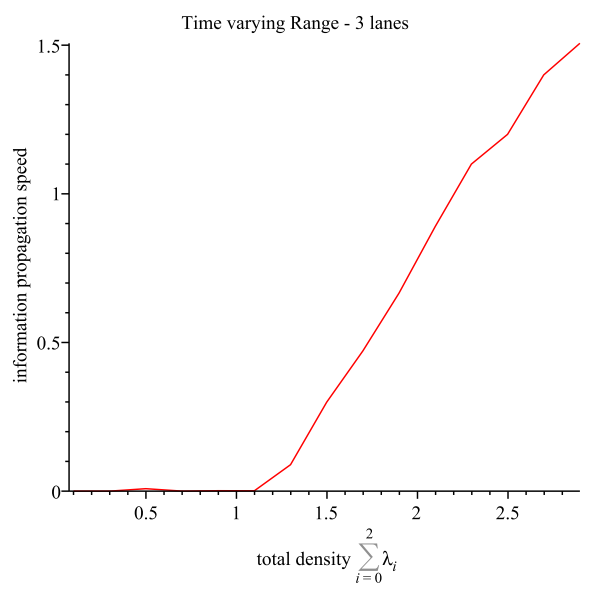

Figure 8: Maple simulations: 3 lanes, varying radio range $\left.\left(P(R>x)=e^{-x^{2}}\right)\right)$. Information propagation speed $v_{p}$ for $\lambda_{0}=\lambda_{1}=\lambda_{2}$, versus the total vehicle density.

radio range per second, 0.5 radio range per second and 0.6 radio range per second. In all figures, we observe the existence of a threshold near $\sum_{i=0}^{i=k} \lambda_{i}=2$, which confirms the analysis: below the threshold the information propagation speed is null, while above the threshold, it increases rapidly.

We then simulate the case of time-varying radio range, and we measure the average information propagation speed. We consider that $P(R>x)=e^{-x^{2}}$, corresponding to the Poisson shot noise model of a one dimensional highway, with path-loss exponent 2. According to our model, vehicles transmit packets of information with Poisson intensity $\nu=5$ transmissions per second. Figures 7 and 8 plot the information propagation speed versus the total vehicle density (with $\lambda_{i}=\lambda_{0}$, for all $i$, and vehicle speeds equal to the corresponding unit-disk simulation speeds), for two and three lanes respectively. Again, we notice the existence of a threshold, where a phase transition occurs concerning the information propagation speed. 


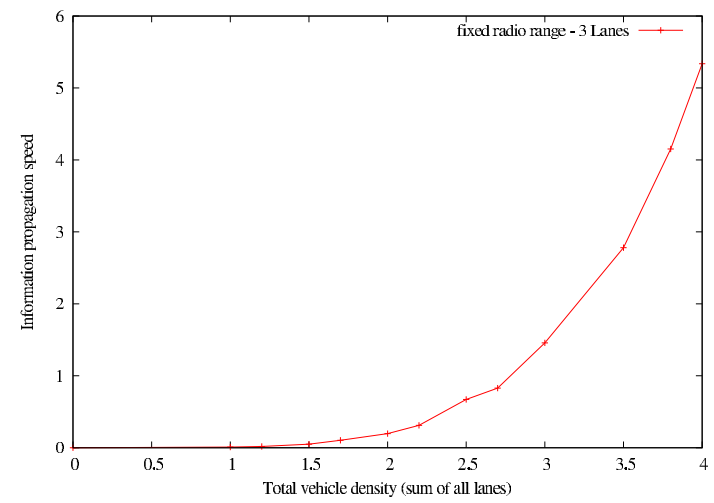

Figure 9: ONE simulations: 3 lanes, fixed radio range. Information propagation speed $v_{p}$ for $\lambda_{0}=\lambda_{1}=\lambda_{2}$, versus the total vehicle density.

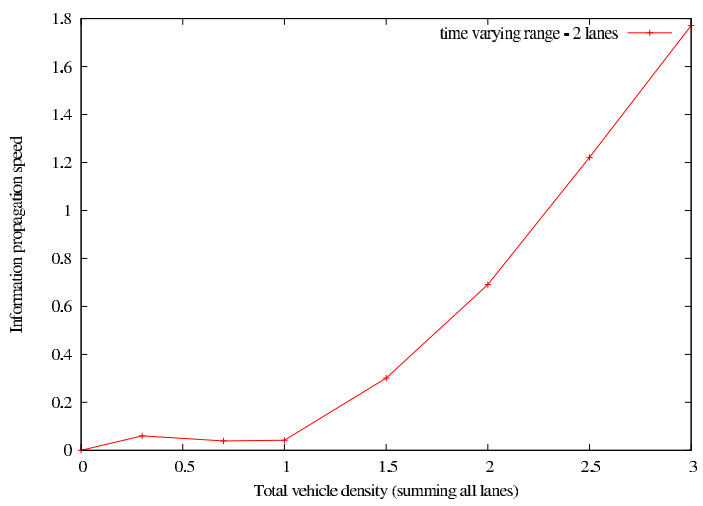

Figure 10: ONE simulations: 2 lanes, varying radio range $\left.\left(P(R>x)=e^{-x^{2}}\right)\right)$. Information propagation speed $v_{p}$ for equal densities in all lanes $\left(\lambda_{0}=\lambda_{1}\right)$, versus the total vehicle density $\lambda_{0}+\lambda_{1}$.

\subsection{ONE Simulations}

We now depart from the exact Poisson model simulations in Maple, and we present simulation results obtained with the Opportunistic Network Environment (ONE [9]) simulator. Vehicles are distributed uniformly on the length of two or more lanes of a road, and move at a constant speed. The total number of vehicles varies from 300 to 4000 . Similarly to the previous section, we measure the fastest possible information propagation speed which is achieved using epidemic broadcast, assuming that radio transmissions are instantaneous and that there are no buffering or congestion delays, with a radio range $R=1$. Again, we vary the vehicle densities $\lambda_{i}$ (vehicles per radio range), and we perform several simulation iterations of randomly generated traffic.

Figure 9 is the equivalent of Figure 5 with corresponding vehicle speeds and densities parameters, for the case of three lanes and a fixed radio range. Figures 10 and 11 correspond respectively to Figures 7 and 8 (for the Maple simulations) for time-varying radio range distributed in $e^{-x^{2}}$, equal lane densities, and with a constant transmission time-slot of 0.2 seconds (instead of Poisson distributed as in Maple). Finally, in Figure 12, we plot the information speed versus the total vehicle density, for the case of a two-lane network, with uneven lane densities (the slow lane 


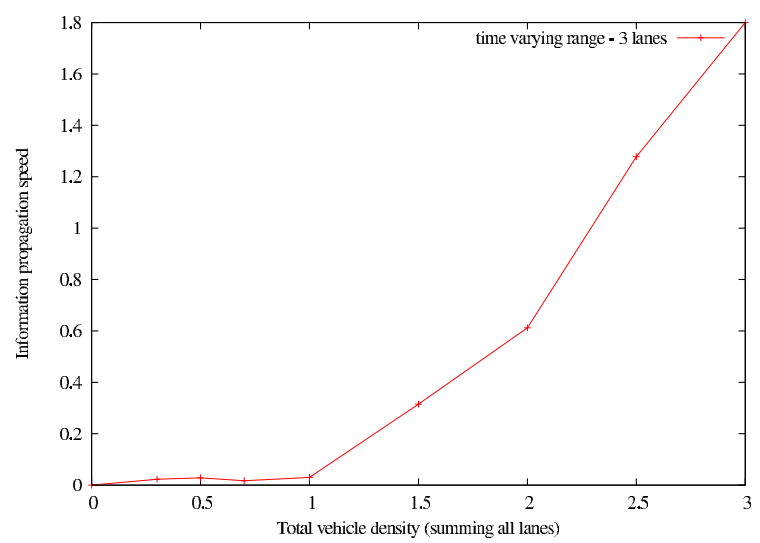

Figure 11: ONE simulations: 3 lanes, varying radio range $\left.\left(P(R>x)=e^{-x^{2}}\right)\right)$. Information propagation speed $v_{p}$ for equal densities in all lanes $\left(\lambda_{0}=\lambda_{1}=\lambda_{2}\right)$, versus the total vehicle density.

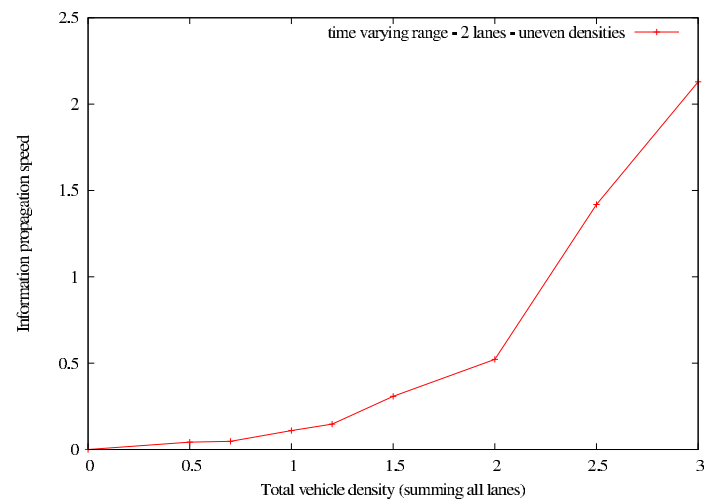

Figure 12: ONE simulations: 2 lanes, varying radio range $\left.\left(P(R>x)=e^{-x^{2}}\right)\right)$. Information propagation speed $v_{p}$ for uneven lane densities $\left(\lambda_{1}=2 \lambda_{0}\right)$, versus the total vehicle density $\lambda_{0}+\lambda_{1}$.

density is twice that of the fast lane). Similarly to what we observed with Maple simulations, we notice in all cases the existence of a threshold, below which the information propagation speed remains almost null, but beyond which it increases dramatically.

\section{Conclusion}

In this paper, we provided a detailed analysis of information propagation speed in multi-lane vehicle-to-vehicle networks. We considered both the case of fixed radio transmission range, and the more realistic case of time-varying radio transmission range. It is indeed paramount to consider variations in radio ranges, since these are heavily experienced in concrete deployments, due to environment versatility and interferences.

We studied networks consisting of multiple lanes of vehicles, varying in speed and in density. For each multi-lane case, we have shown the existence of a density threshold, under which information propagates on average at the speed of the fastest vehicle, and above which information 
propagates significantly faster. We first proved that no such phase transition occurs if there is only one lane, regardless of the density of vehicles, even when one takes into account radio communication range variations at the MAC layer. This result is not, in fact, easy to predict intuitively, because in our model, the radio range $R$ is unbounded, i.e. $P(R>x)>0, \forall x$. We then proved that, on the contrary, for two or more lanes of vehicles with different speeds, a phase transition exists regarding the information propagation speed, when vehicle densities are above a certain threshold. We derived bounds on the corresponding threshold, expressed as a simple relationship between the vehicle density on the fast lane, and the sum of the vehicle densities on the other lanes. We confirmed our analytical results with simulations carried out in several environments (ONE and Maple).

Our model covers a variety of vehicular network scenarios, including one-way and two-way roads, as well as scenarios where road side units and/or parked cars are used as relays. Our methodology seems extendable to the case where the distribution of car speeds may take an infinite number of values between a minimum and a maximum, as this would be equivalent to an infinite number of lanes in our model. A natural further extension, would correspond to the continuous case, where the car speeds may take any value between minimum and maximum. Therefore, these extensions constitute interesting directions for future work.

\section{References}

[1] A. Agarwal, D. Starobinski and T. Little, "Phase Transition of Message Propagation Speed in Delay-Tolerant Vehicular Networks", IEEE Transactions on Intelligent Transportation Systems 13(1): 249-263 (2012).

[2] E. Baccelli, P. Jacquet, B. Mans and G. Rodolakis, "Highway Vehicular Delay Tolerant Networks: Information Propagation Speed Properties". IEEE Transactions on Information Theory 58(3): 1743-1756 (2012).

[3] F. Baccelli, B. Blaszczyszyn and P. Mühlethaler, "An Aloha protocol for multihop mobile wireless networks". IEEE Transactions on Information Theory 52(2): 421-436 (2006).

[4] J. Burgess, B. Gallagher, D. Jensen and B. Levine, "MaxProp: Routing for Vehicle-Based Disruption-Tolerant Networks". In Infocom, 2006.

[5] A. Casteigts, A. Nayak and I. Stojmenovic, "Communication protocols for vehicular ad hoc networks". Wireless Communications and Mobile Computing, 11(5): 567-582 (2011).

[6] F. Dressler, F. Kargl, J. Ott, O. Tonguz and L. Wischhof, "Research challenges in intervehicular communication: lessons of the 2010 Dagstuhl Seminar". IEEE Communications Magazine 49(5): 158-164 (2011).

[7] R. Fracchia and M. Meo, "Analysis and Design of Warning Delivery Service in Inter-vehicular Networks". IEEE Transactions on Mobile Computing. 7(7): 832-845 (2008).

[8] P. Jacquet, B. Mans and G. Rodolakis, "Information propagation speed in mobile and delay tolerant networks", IEEE Transactions on Information Theory 56(10): 5001-5015 (2010).

[9] A. Keranen, J. Ott and T. Karkkainen, "The ONE Simulator for DTN Protocol Evaluation". SIMUTools'09: 2nd International Conference on Simulation Tools and Techniques. Rome, 2009. 
[10] F. Malandrino, C.E. Casetti, C.-F. Chiasserini, C. Sommer and F. Dressler, "Content Downloading in Vehicular Networks: Bringing Parked Cars Into the Picture," In PIMRC 2012.

[11] F. Martelli, M.E. Renda, G. Resta, and P. Santi â "A Measurement-based Study of Beaconing Performance in IEEE 802.11p Vehicular Networks", in Infocom, 2012.

[12] T. Nadeem, P. Shankar, and L. Iftode, "A Comparative Study of Data Dissemination Models for VANETs", in Proc. of MOBIQUITOUS, 2006.

[13] Y. Toor, P. Mühlethaler, A. Laouiti, A. de la Fortelle, "Vehicle ad hoc networks: Applications and related technical issues". IEEE Communications Surveys and Tutorials 10(1-4), pp. $74-88$ (2008)

[14] US-Department of Transportation. National transportation statistics, 2011. US Government printing Office, Washington, DC.

[15] N. Wisitpongphan, F. Bai, P. Mudalige, and O. Tonguz, â "On the Routing Problem in Disconnected Vehicular Ad-hoc Networks", in Infocom, 2007.

[16] H. Wu, R. Fujimoto, and G. Riley, "Analytical Models for Information Propagation in Vehicle-to-Vehicle Networks", in VTCâ Fall, 2004.

[17] S. Yousefi, E. Altman, R. El-Azouzi and M. Fathy, "Analytical Model for Connectivity in Vehicular Ad Hoc Networks". IEEE Transactions on Vehicular Technology, Nov. 2008.

[18] B.X. Wang, T.M. Adams, W. Jin and Q. Meng, "The process of information propagation in a traffic stream with a general vehicle headway: A revisit", Transportation Research Part C, 18 (2010) pp. 367-375.

[19] Z. Zhang, G. Mao and B.D.O. Anderson, "On the Information Propagation Process in Multi-lane Vehicular Ad-hoc Networks", In IEEE ICC 2012.

[20] Z. Zhang, G. Mao and B.D.O. Anderson, "On the Information Propagation Speed in Mobile Vehicular Ad Hoc Networks", In Globecom 2010.

\section{Appendix}

\subsection{Proof of Theorem 3}

We consider that the current car on the fast lane 0 is separated by a gap of length $x$ to the next fast-lane car. Our aim is to evaluate the probability that a chain of cars on the slow lane 1 would bridge the gap, and thus deliver the information to this next car. We will determine when this probability tends to 0 , hence limiting the information propagation to the fast-lane car speed. Therefore, we take the model of the proof of Theorem 1 and apply it to the propagation on lane 1.

In lane 1, when two cars are separated by a gap of length $y$, transmissions are performed within the random space-time vector $(D(y), T(y))$, as defined in the proof of Theorem 1 . When $y$ varies, the propagation of information follows a random walk made of consecutive vectors $(D(y), T(y))$.

Let $f(\zeta, \theta, y)=E\left(e^{-\zeta D(y)-\theta T(y)}\right)$ be the Laplace transform of conditional propagation step $y$. We compute the Laplace transform using (2) and (3). We have, formally:

$$
f(\zeta, \theta, y)=\int_{y}^{\infty} P(R=z) e^{-\zeta z} d z \frac{\nu P(R>y)}{\nu P(R>y)+\theta} .
$$

$\mathrm{RR} \mathrm{n}^{\circ} 8037$ 
The unconditional Laplace transform of the step is $f(\zeta, \theta)=\int_{0}^{\infty} f(\zeta, \theta, y) \lambda_{1} e^{-\lambda_{1} y} d y$. It is defined for all complex $\zeta$, and all complex $\theta$ such that $\Re(\theta) \geq 0$, since for any real $\theta<0, f(\zeta, \theta)$ is singular. We notice that $E(D(y))=\frac{\partial}{\partial \zeta} f(0,0)<\infty$ and $E(T(y))=\frac{\partial}{\partial \theta} f(0,0)=\infty$. We denote $\mathcal{K}$ the kernel of $f(\zeta, \theta)$, i.e., the set of real tuples $(\zeta, \theta)$, such that $f(\zeta, \theta)=1$. This corresponds to a curve in the quarter plane $\zeta \leq 0$ and $\theta \geq 0$, which starts on $(0,0)$, with a zero derivative, since: $\frac{d \theta}{d \zeta}=\frac{\frac{\partial}{\partial \zeta} f(\zeta, \theta)}{\frac{\partial}{\partial \theta} f(\zeta, \theta)}$.

On the referential of lane 0 , the random walk is made of vectors $\left(D(y)-\left(v_{0}-v_{1}\right) T(y), T(y)\right)$, i.e., there is a negative speed drift. Its Laplace step function is $\tilde{f}(\zeta, \theta)=f\left(\zeta, \theta-\left(v_{0}-v_{1}\right) \zeta\right)$. By inversing the Laplace transform, the probability density $p(x, t)$, that a chain of relays on lane 1 reaches the distance $x$ at time $t$, satisfies:

$$
p(x, t) \leq \frac{1}{(2 i \pi)^{2}} \iint_{\Re(\zeta)=\zeta_{0}, \Re(\theta)=\theta_{1}} \frac{\tilde{f}(\zeta, \theta)}{1-\tilde{f}(\zeta, \theta)} e^{\zeta x+\theta t} d \zeta d \theta,
$$

where $\frac{\tilde{f}(\zeta, \theta)}{1-\tilde{f}(\zeta, \theta)}=\sum_{n \geq 1} \tilde{f}(\zeta, \theta)^{n}$ is the Laplace transform of a non-null sequence of steps. This is an upper bound on $p(x, t)$, since a chain can hit the position $x$ several times. The pair $\left(\zeta_{0}, \theta_{1}\right)$ belongs to the definition domain of $\frac{\tilde{f}(\zeta, \theta)}{1-\tilde{f}(\zeta, \theta)}$, i.e., there exists $\theta_{0}$, such that $\theta_{1}>\theta_{0}$ and $\left(\zeta_{0}, \theta_{0}-\left(v_{0}-v_{1}\right) \zeta_{0}\right) \in \mathcal{K}$.

Since the curve of $\mathcal{K}$ has zero slope at point $(\zeta, \theta)=(0,0)$, and since $v_{0}-v_{1}>0$, there exist non-positive $\zeta_{0}$ and $\theta_{0}$, and thus $\theta_{1}$. Therefore, we can compute the probability $q(x)$ that a chain of relays on lane 1 bridges the gap $x$ (for any time $t$ ):

$$
\begin{aligned}
q(x) & =\int_{x}^{\infty} \int_{0}^{\infty} p(z, t) d z d t \\
& \leq \frac{1}{(2 i \pi)^{2}} \iint_{\Re(\zeta)=\zeta_{0}, \Re(\theta)=\theta_{1}} \frac{\tilde{f}(\zeta, \theta)}{1-\tilde{f}(\zeta, \theta)} \frac{e^{\zeta x}}{\zeta \theta} d \zeta d \theta \\
& =O\left(e^{\zeta_{0} x}\right) .
\end{aligned}
$$

The quantity $q(x)$ is an upper bound of the probability that a car on lane 1 starts a chain of relays that will bridge a gap of length $x$. Therefore, the average time needed to find a car that starts such a chain is $\frac{1}{\left(v_{0}-v_{1}\right) \lambda_{1} q(x)}=\Omega\left(e^{-\zeta_{0} x}\right)$. When $x$ varies, the average time is infinite as soon as $\lambda_{0}<-\zeta_{0}$ (recall that $\zeta_{0}$ is non-positive). Therefore, the theorem is proved, as an infinite average time to bridge the gap implies an information propagation speed restricted to the car speed.

\subsection{Proof of Theorem 5}

Due to space constraints, we give a sketch of the proof. Let $j$ be an integer between 1 and $k$. We work again with the upper bound scheme developed for the proofs of Theorems 1 and 3 . Let $x$ a gap between a car on lane $j$ and the next car on the same lane. We denote $D_{j}(x)$ and $T_{j}(x)$, respectively, the distance traveled by the message and the time needed for this distance to bridge the gap $x$, by taking relays on slower lanes only (i.e. on lane $j+1$ and further). Therefore, by considering the part of the highway with lanes $i \geq j$ only, we equivalently have $D_{k}(x)=D(x)$ and $T_{k}(x)=T(x)$.

We define the Laplace transforms $f_{j}(\zeta, \theta, x)=E\left(e^{-\zeta D_{j}(x)-\theta T_{j}(x)}\right)$ and $f_{j}(\zeta, \theta)=\int_{0}^{\infty} f_{j}(\zeta, \theta, x) \lambda_{j} e^{-\lambda_{j} x} d x$. Our aim is to prove by recursion on decreasing lane indexes that there exist $\zeta_{j}<0$ and $\alpha_{j}>0$, such that: 
- $f_{j}(\zeta, \theta)$ is defined for all complex tuples $(\zeta, \theta)$, such that $\Re(\zeta)>\zeta_{k}$ and $\Re(\theta) \geq 0$;

- $E\left(T_{j}(x)\right)=O\left(e^{\alpha_{j} x}\right)$.

The first point easily comes from the fact that $\left|f_{j}(\zeta, \theta)\right| \leq f_{j}(\Re(\zeta), \Re(\theta))$, and since $D_{j}(x) \leq x+\bar{R}$ in probability, for all $\zeta<0$ and $\theta>0: f_{j}(\zeta, \theta) \leq e^{-x \zeta} g(\zeta)$. Thus, $f_{j}(\zeta, \theta) \leq \frac{\lambda_{j}}{\lambda_{j}+\zeta} g(\zeta)$ and we can choose $\zeta_{j}=-\lambda_{j}$.

The second point is more delicate. Taking the framework of the proof of Theorem 3, we define $q_{j}(x)$ as the probability that a chain of relays on lane $i+1$ bridges a gap of length $x$ on lane $i$. Therefore, we have the inequality, following (4),

$$
q_{j}(x) \leq \frac{1}{(2 i \pi)^{2}} \iint_{\Re(\zeta)=\zeta_{0, j}, \Re(\theta)=\theta_{1, j}} \frac{\tilde{f}_{j}(\zeta, \theta)}{1-\tilde{f}_{j}(\zeta, \theta)} \frac{e^{\zeta x} d \zeta d \theta}{\zeta \theta},
$$

for $\tilde{f}_{j}(\zeta, \theta)=f_{j+1}\left(\zeta, \theta-\left(v_{j}-v_{j+1}\right) \zeta\right)$, and $\left(\zeta_{j, 0}, \theta_{1, j}\right)$ in the definition domain of $\frac{\tilde{f}_{j}(\zeta, \theta)}{1-\tilde{f}_{j}(\zeta, \theta)}$, i.e. $\left|\tilde{f}_{j}\left(\zeta_{0, j}, \theta_{1, j}\right)\right|<1$, with $\zeta_{0, j}<0$ and $\theta_{1, j}<0$.

Such values exist for the following reasons:

1. The function $f_{j+1}(\zeta, \theta)$ is defined for all $\theta>0$ and $\zeta>\zeta_{j}$. Thus, $\tilde{f}_{j}(\zeta, \theta)$ is defined for all $\theta>\left(v_{j}-v_{j+1}\right) \zeta$.

2. The kernel $\mathcal{K}_{j+1}$ of function $f_{j+1}(\zeta, \theta)$ is defined for $\zeta<0, \theta>0$ and $\frac{d \theta}{d \zeta}=0$, if we assume that $\lambda_{j+1}<\alpha_{j+1}\left(\right.$ since $\left.\int_{0}^{\infty} E\left(T_{j+1}(x)\right) \lambda_{j+1} e^{-\lambda_{j+1}} d x=\infty\right)$. Thus, there exist $\zeta_{0, j}<0$ and $\theta_{0, j}<0$, such that $\left(\zeta_{0, j}, \theta_{0, j}-\left(v_{j}-v_{j+1}\right) \zeta_{0, j}\right) \in \mathcal{K}_{j+1}$.

3. We take $0>\theta_{1, j}>\theta_{0, j}$.

Therefore, $q_{j}(x)=O\left(e^{\zeta_{0, j} x}\right)$ and, consequently, $E\left(T_{j}(x)\right) \geq \frac{1}{q_{j}(x)}=\Omega\left(e^{-\zeta_{0, j} x}\right)$. Equivalently to Theorem 3 , this implies that information propagation on lane $j$ is restricted to the car speed. We conclude the proof by recursion on $j$, descending down to lane 0 . 


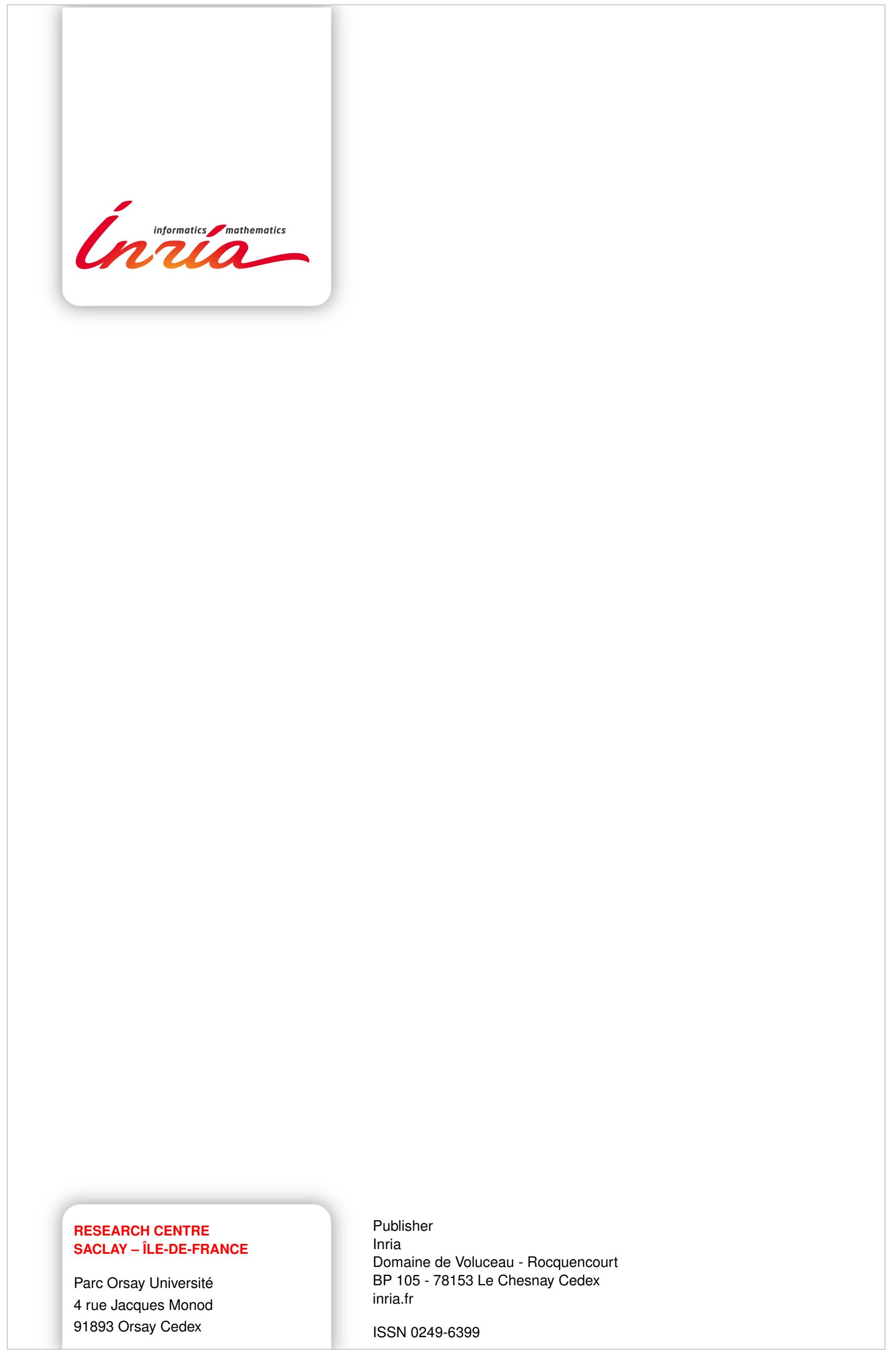

